\title{
Designing and implementation of an intelligent manufacturing system
}

\author{
Michael Peschl ${ }^{1}$, Norbert Link ${ }^{2}$, Michael Hoffmeister ${ }^{3}$, Gil Gonçalves ${ }^{4}$, Fernando L.F. \\ Almeida ${ }^{5}$ \\ ${ }^{1}$ Harms \& Wende GmgH, ${ }^{2}$ University of Applied sciences Karlsrube, LAF, ${ }^{3}$ Manufacturing Engineering \\ and Automation Fraunbofer Institute (GERMANY) \\ ${ }^{4}$ Department of Informatics of University of Porto, ${ }^{5}$ Faculty of Engineering of University of Porto \\ (PORTUGAL) \\ michael.peschl@,hwh-k.arlsmbe.de,norbert.link@,hs-k.arlsrube.de,boffmeister@ipa.fraunhofer.de, \\ gil@,fe.up.pt, almd@fe.up.pt
}

Received June 2011

Accepted September 2011

\section{Abstract:}

Purpose: The goal of XPRESS is to establish a breakthrough for the factory of the future with a new flexible production concept based on the generic idea of "specialized intelligent process units" ("Manufactrons") integrated in cross-sectoral learning networks for a customized production. XPRESS meets the challenge to integrate intelligence and flexibility at the "highest" level of the production control system as well as at the "lowest" level of the singular machine.

Design/methodology/approach: Architecture of a manufactronic networked factory is presented, making it possible to generate particular manufactrons for the specific tasks, based on the automatic analysis of its required features.

Findings: The manufactronic factory concept meets the challenge to integrate intelligence and flexibility at the "highest" level of the production control system as well as at the "lowest" level of the singular machine. The quality assurance system provided a $100 \%$ inline quality monitoring, destructive costs reduced $30 \%-49 \%$, 
the ramp-up time for the set-up of production lines decreased up to $50 \%$ and the changeover time decreased up to $80 \%$.

Research limitations/implications: Specific features of the designed manufactronic architecture, namely the transport manufactrons, have been tested as separate mechanisms which can be merged into the final comprehensive at a later stage.

Practical implications: This concept is demonstrated in the automotive and aeronautics industries, but can be easily transferred to nearly all production processes. Using the manufactronic approach, industrial players will be able to anticipate and to respond to rapidly changing consumer needs, producing highquality products in adequate quantities while reducing costs.

Originality/value: Assembly units composed of manufactrons can flexibly perform varying types of complex tasks, whereas today this is limited to a few predefined tasks. Additionally, radical innovations of the manufactronic networked factory include the knowledge and responsibility segregation and trans-sectoral process learning in specialist knowledge networks.

Keywords: intelligent manufacturing, production units, quality models, industrial workflow models

\section{Introduction}

\subsection{The concept of intelligent manufacturing systems}

Global competition and rapidly changing customer requirements are forcing major changes in the production styles and configuration of manufacturing organizations. Increasingly, traditional centralized and sequential manufacturing process planning, scheduling, and control mechanisms are being found insufficiently flexible to respond to changing production styles and high-mix low-volume production environments (Shen et al., 1999). The traditional approaches limit the expandability and reconfigurability of the manufacturing systems (Sanchez \& Nagly, 2001). The centralized hierarchical organization may also result in much of the system being shut down by a single point of failure, as well as plan fragility and increased response overheads (Yang \& Xue, 2003). 
In the last twenty years manufacturing concepts have had several redefinitions. In the eighties, the concept of flexible manufacturing systems (FMC) was introduced to develop a new family of products with similar dimensions and constraints, but nowadays, the capacity of reconfiguration has become a major issue for improving the functioning of industrial processes (Revilla et al., 2008). Indeed, today a main objective is to adapt quickly in order to start a new production or to react in a failure occurrence. Intelligent manufacturing systems (IMS) offer not only both flexibility and reconfigurability, but also this concept brings more than a few ideas of software intelligence meanings, which contemplated characteristics such as autonomy, decentralization, flexibility, reliability, efficiency, learning, and selfregeneration (Revilla et al., 2008; Mekid et al., 2009; Shen et al., 2006).

The current challenge is to develop collaborative and reconfigurable manufacturing control systems that support efficiently small batches, product diversity, high quality and low costs, by introducing innovative characteristics of adaptation, agility and modularization. Information and communication technologies, and artificial intelligence techniques, have been used for more than two decades addressing this challenge. Namely, agent-based and Holonic manufacturing control seem to be suitable to face these requirements such as modularity, scalability, autonomy and re-usability, since they present decentralization of control over distributed structures. When properly designed and implemented, agent-based control systems result in a performance that is flexible, robust, adaptive and fully tolerant, which are key factors for manufacturing success in the increasingly global marketplace (Aized, 2010).

Recently, there has been growing interest in the holonic approach to the development of complex industrial and business systems. Motivated by the need to enable these man-made systems to adapt to disturbances while maintaining system stability and efficient use of resources, Holonic systems were inspired by Arhtur Koestler's early observations of the structure and behavior of living organisms and social organizations (Koestler, 1967). Like multi-agent systems (MAS), holonic systems are composed of self-reliant units that are capable of flexible behavior. More specifically though, a holon can be thought of as a special type of agent that is characteristically autonomous, cooperative and recursive, that populates a system where there is no high-level distinction between hardware and software. Although both approaches share many basic concepts, research in each area has been conducted independently for the most part. Holonic systems research has primarily focused on intelligent manufacturing systems and has been organized around the international Holonic Manufacturing Systems (HMS) consortium (Cheng 
et al., 2004). In contrast, MAS research is much broader in scope, focusing generally on the development of systems in which "data, control, expertise or resources are distributed; agents provide a natural metaphor for delivering system functionality; or a number of legacy systems must be made to interwork" (Leitão, 2009). The manufacturing enterprises of the 21st century are in an environment where markets are frequently shifting, new technologies are continuously emerging, and competition is globally increasing. Manufacturing strategies should therefore shift to support global competitiveness, new product innovation and customization, and rapid market responsiveness (Prajogo et al., 2007). The next generation manufacturing systems will thus be more strongly time-oriented (or highly responsive), while still focusing on cost and quality. Such manufacturing systems will need to satisfy a number of fundamental requirements, including (Shen et al., 2006; Chituc \& Restive, 2009):

- Full integration of heterogeneous software and hardware systems within an enterprise, a virtual enterprise, or across a supply chain

- Open system architecture to accommodate new subsystems (software or hardware) or dismantle existing subsystems "on the fly"

- Efficient and effective communication and cooperation among departments within an enterprise and among enterprises

- Embodiment of human factors into manufacturing systems

- Quick response to external order changes and unexpected disturbances from both internal and external manufacturing environments

- Full tolerance both at the system level and at the subsystem level so as to detect and recover from system failures and minimize their impacts on the workflow environment

\subsection{The XPRESS approach}

The EU project XPRESS (IP026674-2) aims at developing a concept of an IMS and introduces a completely new scalable concept of a manufactronic networked factory, which is composed by a coordinated team of specialized autonomous entities (manufactrons), each knowing how to do a certain process optimally. Manufactrons encapsulates the different functionalities within a factory. By doing so, a single manufactron is able to perforem the assigned tasks optimally within linked networks by considering their knowledge. Each manufactron has mechanisms 
of self-learning, self-organization, and knowledge acquisition (drawn by experience). This knowledge based concept integrated the complete process chain, from the production planning to the assembly, the quality assurance of the produced/assembled products and the reusability of process units (Peschl, 2010).

The new concept of Manufactronic networked factory is developed and demonstrated by a strong industry-lead partnership in order to meet the still remaining industrial needs with regard to:

- Production configuration and simulation - XPRESS intends to significantly decrease the ramp-up time for assembly lines, increase the reusability of assembly components and optimize the entire of the assembly process

- Manufactron guided production flow - for the assembly and manufacturing of different types and variable volumes of products on a single flexible line and achievement of a high level of reusability

- Manufactronic machines and human integration - a) reducing the effort needed for setting up a single process; b) providing most efficient and reliable inline quality assurance systems for the process; c) reacting intelligently on disturbances; d) providing a factory-wide process monitoring systems; e) allowing the reuse of disassembled components

The work report in this paper proposes a completely new scalable concept of a manufactronic networked factory. The central goal of XPRESS is to achieve a breakthrough for the knowledge-based and agile manufacturing enterprise of the future (EC, 2004) with an innovative flexible and fast reconfigurable manufacturing solution based on the generic idea of the "Manufactronic networked factory". XPRESS takes the whole production process into consideration in which the machines are not only communicating with each other but are members of a coordinated team of specialized autonomous objects (Manufactrons) in learning networks (environment of intelligent collaboration).

The remainder of this paper is organized as follows: In section 2 we present the standard structure of a Manufactron. Section 3 describes the concept of a Manufactronic networked factory giving an overview of its components. Section 4 describes the implemented approach followed by the project. Section 5 presents the main results obtained by the project, particularly related to the three demonstrated scenarios. Finally, the conclusion of our work is drawn and an outlook for further work is given in section 6 . 


\section{The manufactron concept}

A Manufactron is a self-contained entity, which is encapsulating expertise and functionality and interacts with its environment by the exchange of standardized synchronous messages. This notion of Manufactron can be better understood looking for the four different views presented in Figure 1.

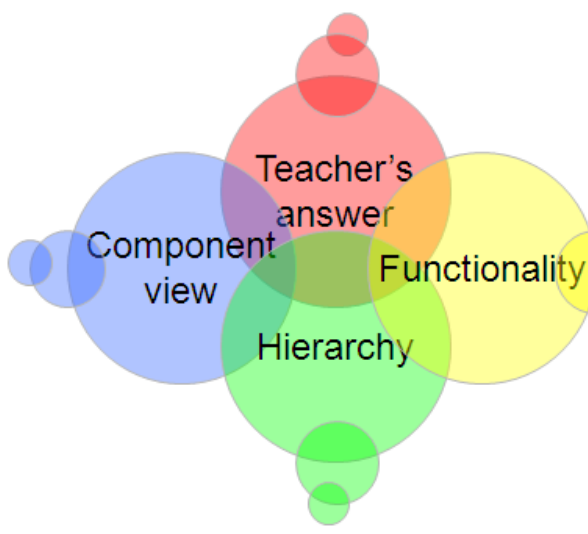

Figure 1. Different perspectives for manufactron definition

The component view lists several components, which shall be part of every "typical" Manufactron. These components can be implemented into a library, the "Manufactronic framework", in order to re-use the same components for nearly every Manufactron. Nonetheless, this is not mandatory. If a Manufactron realizes its own components, which are only behaving in the same way, it will comply also to the definition of a "Manufactron".

The functionality view gives an answer, which functionality has to be realized by a piece of software or order to name it "Manufactron". Therefore again, the "Manufactron" may rely to its own implementation, if only it's realizing the needed functionality to be called a "Manufactron".

The hierarchy view proposes a set of three different levels (Production Configuration Manufactrons, Workflow/Quality Manager Manufactrons and Production Manufactrons), on which artifacts of the XPRESS project shall be realized. Every Manufactron shall fit into exactly one of these levels, where the first and second do have some special restrictions and responsibility. It will be therefore expected, that most of custom-implemented Manufactrons will reside on the level of "Production Manufactrons".

The Manufactron shall be self-contained. It is expected that a typical Manufactron may be added to a Manufactronic factory by just plugging an additional device into 
the factory's network. Therefore, the Manufactron shall be realized as an independent piece of implementation rather than a very distributed entity, where a lot of different fractions of the entity are to be integrated into different systems of the factory, as to be the Enterprise Resource Planning (ERP) and the Manufacturing Execution Systems (MES) system of different kinds of Programmable Logic Controller (PLC) systems (Ribeiro \& Gonçalves, 2010).

The Manufactron shall not only realize a simple functionality, but shall also provide expertise on this functionality to the outer world. This allows the outer world to state a task to be fulfilled to the Manufactron without the need to know about every small detail associated with these tasks. The encapsulation of expertise is therefore the answer to demands stated by multi-variant production (higher levels do not have to concern about small details) and flexibility in terms of production resources (a task is not depending on a very special welding machine, but can be understood by every welding machine).

The Manufactrons are agents that decide how to reach their given goals best, but not when to do it. The task execution is triggered from outside as defined by another Manufactron category, named "workflow manager" overlooking the factory level with dedicated knowledge expertise (Almeida et al., 2010). This results in a Manufactron hierarchy:

- Field level: "Production Manufactrons" (executing basic manufacturing tasks) and "Super Manufactrons" (co-ordinating groups of Production Manufactrons)

- Factory level: "Workflow managers" (controlling the production flow of an item) conforming the manufacturing execution system up to production planning

- Bureau level: "Configuration Manufactrons" responsible for finding an optimum production configuration and for the creation of workflow managers for different product variants or for varying production conditions

The capabilities of a Manufactron are described in the Manufactron Self Description (MSD) document. Each Manufactron or other entity in the Manufactronic factory can request the MSD of a Manufactron. The main information contained in a MSD file include the information on the capabilities of the Manufactron, the information regarding the task description, and the quality result items generated by the Manufactron after the execution of a task. 


\section{Manufactronic networked factory}

A high challenge of the XPRESS specification and development work is the interaction of the different components of the whole system. The communication scheme between components of the different layers (ERP, shop floor and cell level) and also within the layers must be powerful, flexible and extensible. A main focus of the specification in this area was to develop a uniform and standardized communication protocol for the Manufactronic Framework. For that purpose, a XML based approach has been chosen, which guarantee a very flexible and extensible system, being at the same time powerful enough to handle all data and signals to be transported between system components.

The basic approach of the manufactronic communication scheme is a synchronous exchange of documents. For that, only two types of documents do exist:

- $\quad$ Task description documents (TDD)

- Quality result documents (QRD)

TDDs provide input information for a Manufactron. This document includes all information needed by the Manufactron to perform a task. This includes the information, what to be done, the task goals as well as specific boundary conditions for task performing (Pollak et al., 2010). The information in the TDD is a XML-based language and has hierarchical structure. On the other side, QRDs are released by the Manufactrons after they received a TDD and performed the task. QRDs do not only contain quality information (as the name might suggest). It contains any kind of data, which is the result of performing a task.

The network topology of the manufactronic networked factory is presented in the sections below.

\subsection{Production configuration system}

The Production Configuration System (PCS) is the component responsible for the simulation process, execution start and execution workflow management. During the simulation process or planning phase, its core tasks include the definition of the optimal configurations based on product's definition, processes and production goals. After finding the best production configurations, the PCS is able to issue production orders by instantiating Workflow Managers, which control all the production process in the lower level layers. This is called the production phase. If a problem occurs during this phase, the PCS is able to find a sub-optimal 
configuration to be applied to the production process. Figure 2 presents the hierarchy of the complete system deployed on the factory.

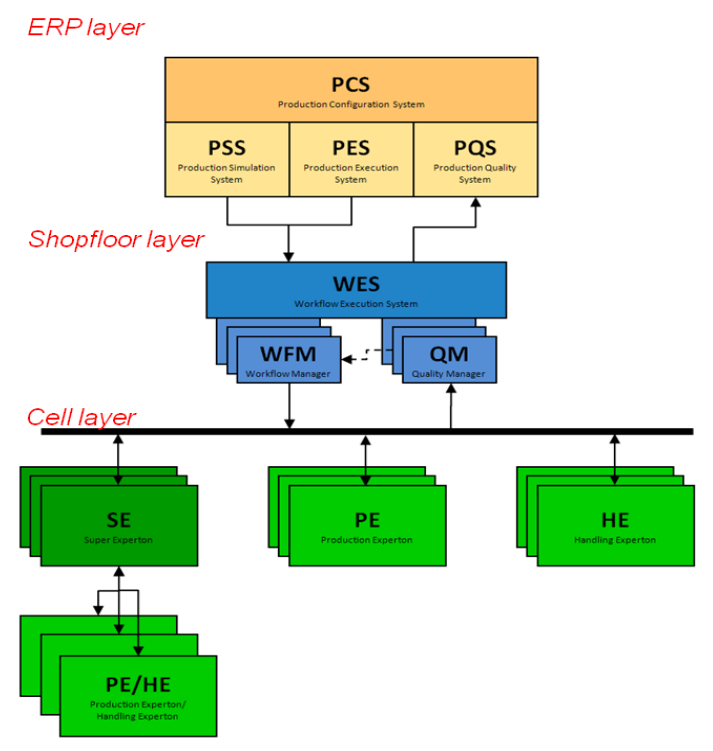

Figure 2. Overview of the manufactronic architecture (Almeida et al., 2010)

The system is comprised of the PCS, which is the main subject of this document, the Workflow Execution System (WES), and the lower level Manufactrons: Super Manufactron, Production Manufactron, Human Manufactron and Handling Manufactron. The WES, instantiated by the PCS during the simulation phase or production phase, is comprised of Workflow Manager (WFM) and Quality Manager (QM) components. This component, the WES, is the mediator between the PCS and all the other Production Manufactrons (PMs) or Handling Manufactrons (HMs) or Super Manufactrons (SMs). Each started instance of WFM or QM is responsible for the control and organization of the Manufactrons underneath it. This allows the WES to suspend or to persist the Manufactrons, if no activity is to be performed. It is the responsibility of every Manufactron to communicate with dependent or superior Manufactrons (SMs or WES "Manufactron"). As far as the communication goes, it is done along with the arrows depicted in the figure, representing the exchange of XML data within the system. The system's communication is synchronous, therefore, each TDD sent to a manufactron must return a QRD. In case that the operation is not performed, a QRD containing an error message must be sent to upper level.

The PCS is divided in three components: Production Simulation System (PSS), Production Execution System (PES), and finally Production Quality System (PQS). Each sub-component has its own components, in order to make PCS implementation easier to maintain. The PSS performs simulation tasks, using 
different workflows with various Production Manufactrons and configurations. On the other hand, the PES is responsible for receiving and selecting the best configuration from production jobs issued by external ordering systems, such as SAP. Regarding PQS, this component is responsible for storing and retrieving the quality results in XML formatted files denominated Quality Result Documents (QRDs), which are generated at the end of the production cycle and contain the complete quality information of the entire production process and the product itself.

\subsection{Distributed workflow execution system}

Originally the Manufactronic system specification supports only a single Workflow Execution System (WES). This initial limitation introduced some disadvantages, turning impossible the support for parallelism on lower levels. In fact, Manufactrons that received a TDD are required to finish their task and answer with a QRD, before the next TDD can be sent. While this synchronous behavior reduces system complexity, it prevents simple implementations for pipelined machines. Pipelined machines can start production of a second product, before the first product is finished. Depending on the size of the pipeline, $n$ products can be started during the production time of a product.

To mitigate these disadvantages, the concept of a "distributed WES" is introduced. The central factory WES can optionally be assisted by one or more local Sub-WES systems. The Sub-WES can be integrated as part of a machine (hence the term "local"). Its task is to execute workflows locally. Figure 3 illustrates the distributed WES approach.

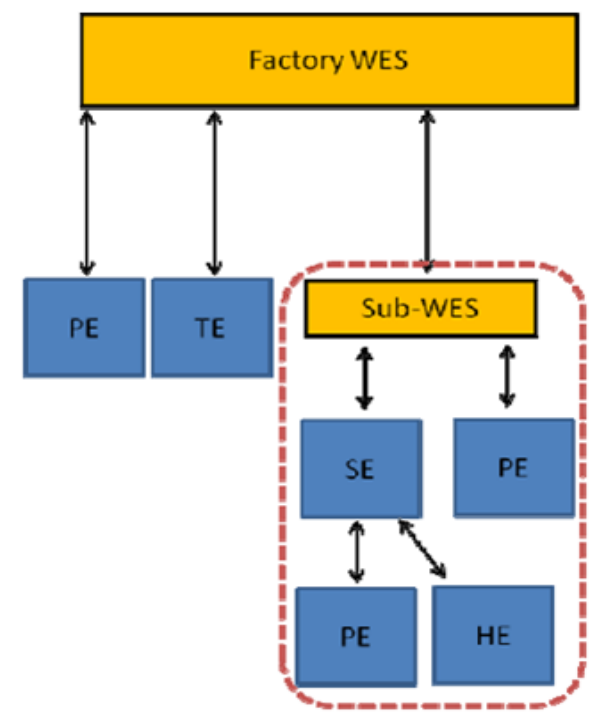

Figure 3. Distributed WES 
One property of the WES is that it can keep track of multiple workflows concurrently, by instantiating Workflow Managers for each of them. This property solves the parallelism problem in pipelined machines, as the Sub-WES can instantiate a WFM for each product in the pipeline.

Because the Sub-WES is dedicated to a single machine, its workload is more predictable, and communication links between Manufactrons and Sub-WES remain local. The delay that is introduced by the WES is therefore much more predictable. Up to a certain extend it is even controllable, by selecting computing and communication hardware to match the machine's required performance.

Furthermore, the Sub-WES contributes to the robustness of the system. If the Factory WES is unable to issue TDDs, or if the communication infrastructure to the machine fails, the Sub-WES can be instructed to locally re-issue the last TDD(s) repeatedly. This way a fall-back option is created, the machine can continue producing, even when it is offline.

\subsection{Directory service}

The Directory Service (DS) is a required component in the Manufactronic communication framework. It has a supporting role in all communication transactions between the Manufactronic components. The DS provides services to register and resolve network addresses and Manufactron names. Furthermore, it provides authentication and security services to the communicating parties.

The DS is not a manufactron and has a special interface to be called. The existence of this component brings relevant advantages to the Manufactronic networked architecture in terms of robustness, tolerance of intermittent network errors, fast reaction to failures and a reliable messaging system.

The DS stores every change in a persistent storage using XML. The Manufactrons are identified by an unique name and a Global Unique Identifier (GUID). Besides that, DS has a ping process, which in regular intervals makes sure whether the registered manufactrons are alive. After Directory Service starts, it reads data from the persistent storage (if not exists creates an initial repository). It registers all the manufactrons in the Ping process (regardless if the status is ALIVE or UNAVAILABLE) and starts the process. If a node doesn't answer to a Ping request or a different manufactron answers from the registered endpoint, it is automatically tagged as UNAVAILABLE. An UNAVAILABLE manufactron is removed from the DS 
after a configurable tolerance time. On the other side, if a node answers to a Ping request and its STATUS was UNAVAILABLE, it is tagged as ALIVE back again.

Figure 4 depicts the Directory Service interface of the service.

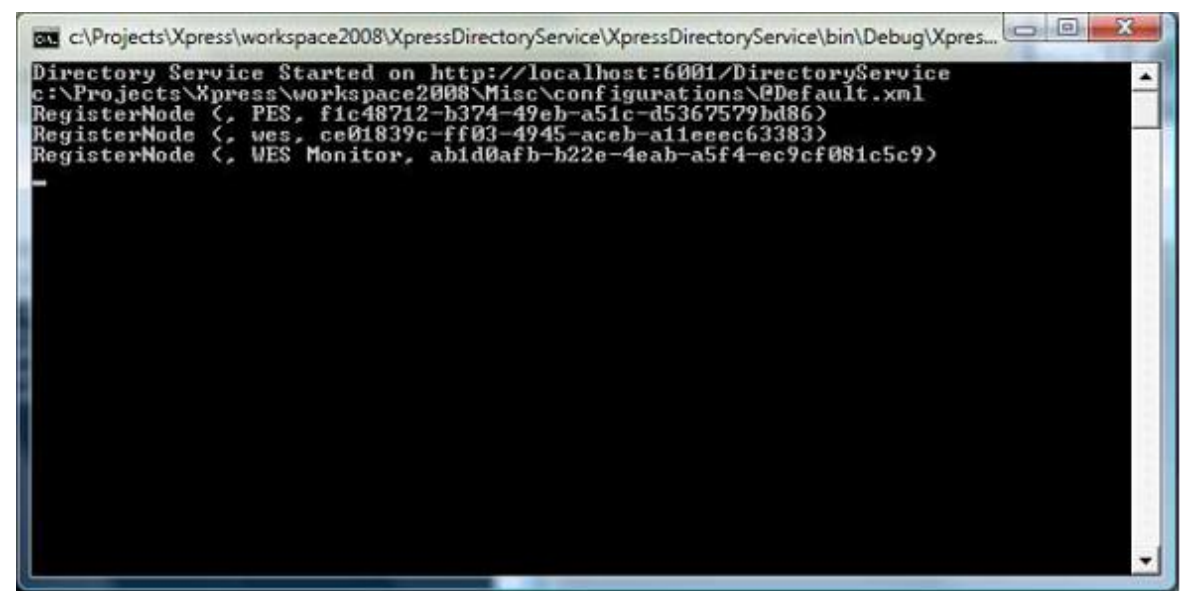

Figure 4. Directory Service interface

\subsection{Monitoring service}

Monitoring Service (MS) is a kind of Supervisory Control and Data Acquisition (SCADA) service, which is intended to show an overview of the manufactronic factory. It dynamically displays the so-called "widgets", which is maintained by individual manufactrons. MS uses Windows Presentation Foundation (WPF) as a user interface technology. WPF is an XML-based language, which makes it very suitable to realize a SCADA-like system.

MS puts additional graphical elements to these widgets such as tracking products. Every product has a unique id, such as RFID or barcode during the production, so it can display the whereabouts of the products. MS service has a logging facility, which can show what is happening in the factory. Analyzing this log can provide valuable information for eliminate network errors.

MS is tightly integrated with the Directory Service. The registration of manufactrons in the Monitoring Service is completely automatic. MS monitors DS for changes in the manufactronic hierarchy. This is based on Manufactrons' status, created and updated time. If a manufactron is temporary unavailable (e.g. intermittent network failure) the widget's border becomes red on the MS canvas. After the manufactron is removed from the DS, it is removed from the MS as well.

When MS realizes that a new manufactron registered in the DS, it sends a subscription request directly to the manufactron. The manufactron registers this in 
its local subscription list, sends a widget template and the initial data to the MS. The manufactron appears immediately on the MS canvas. From now on the Manufactron notifies MS of every changes of its status. Although the communication is not real-time, it is close to it. The notification messages frequency can be very high, so it can happen, that the messages arrive in a different order, than they were sent. To solve this, MS just drop those messages, which are were sent earlier, than the last received message. A sequence number by manufactron intends to handle this issue. Besides that, as the Monitoring Service is also a Manufactron, it is capable of intervening the execution of the workflow, such as terminating the execution and dropping the product. Although this service only displays the widgets at the moment, it has the potential to become a more powerful controller.

Figure 5 depicts the Monitoring Service interface of the service.

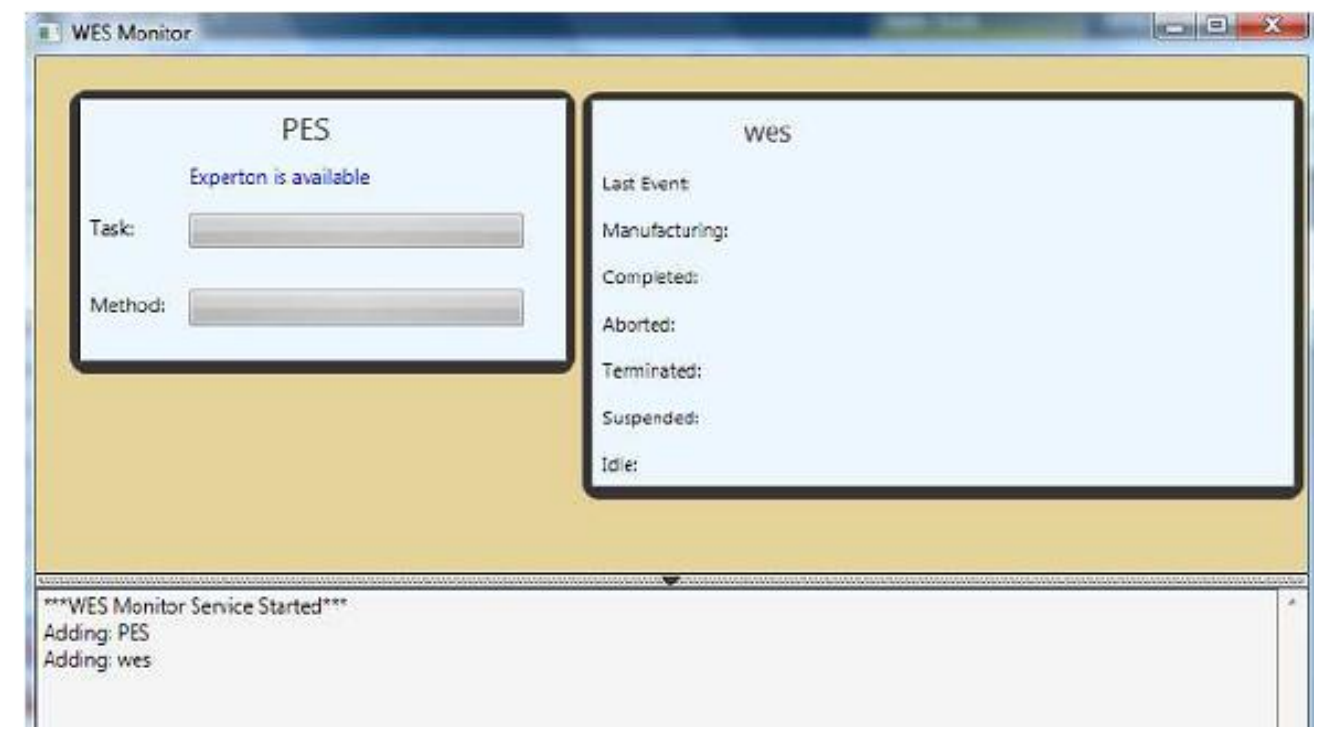

Figure 5. Monitoring Service interface

\section{5 "Factory floor" manufactrons}

"Factory floor" Manufactrons are the manufactrons that can be found on the factory floor, like the Production Manufactrons, Handling Manufactrons, Transport Manufactrons and Sub-WES.

The Production Manufactron is responsible to perform a task at the shop-floor and implements process knowledge and/or connections to the filed level. Handling Manufactron is a special case of a Production Manufactron that is responsible to handily manipulate a work-piece. Transport Manufactron is responsible to transport a work-piece on a factory floor (the XPRESS supports two kinds of transportations: based on conveyor pallets and AGVs). Finally, the sub-WES acts as an unit 
coordinator realizing the workflow and quality manager attached to a single product.

In most, if not all cases, the Manufactron will be communicating with its associated production system, like a PLC system, a weld controller, a robot system or the controls of a vehicle. This communication may be based on 100BASE-TX Ethernet, but other standards or proprietary interfaces are also allowed.

The availability requirements for these Manufactrons are less demanding, compared to the PCS/PQS, WES and DS, as a failure of one of these components will not lead to a standstill of the complete factory.

The amount of processing power needed is greatly dependent on the type of Manufactron and its implementation. If processing power allows, it is possible and allowed to run multiple Manufactrons on one piece of hardware.

\subsection{Human-machine interface}

Figure 6 illustrates the Production Execution System (PES) in its diagram form, where the Workflow Manager (WFM) object is instantiated through the Workflow Execution System interface by issuing a TDD, which is forwarded by the WFM to a Human Handling Manufactron and, simultaneously, to a Welding Manufactron. Both Manufactrons together perform a row spot welding task on a car door. The generated quality data is sent back to the Quality Manager of the WFM, in QRD format. This Quality Manager assesses the overall quality of each task and their combination and then sends it back to the PCS, where the quality results are displayed to the end user.

The PES provides a Graphical User Interface (GUI) that simplifies the end user's interaction with the available PES functionalities. Among all the available functionalities is worth to note the loading of XML files with TDD/QRD library, generation of workflow managers (WFM) and Quality Managers (QM), interface to WES and displaying quality results.

At start-up, the end user is offered an interface where it is possible to load a specific TDD and set the number of executions for the chosen task. After the user starts the PCS execution, a Workflow Manager (WFM) object is instantiated and the loaded TDD is forwarded to this new object. This object will handle the task description to the lower level Manufactrons which will perform the task described in the TDD, while the WFM is controlling the lower level Manufactronic Layer by 
updating the workflow status of each activity. The GUI is able to show this process at run-time. This situation is illustrated in Figure 7.

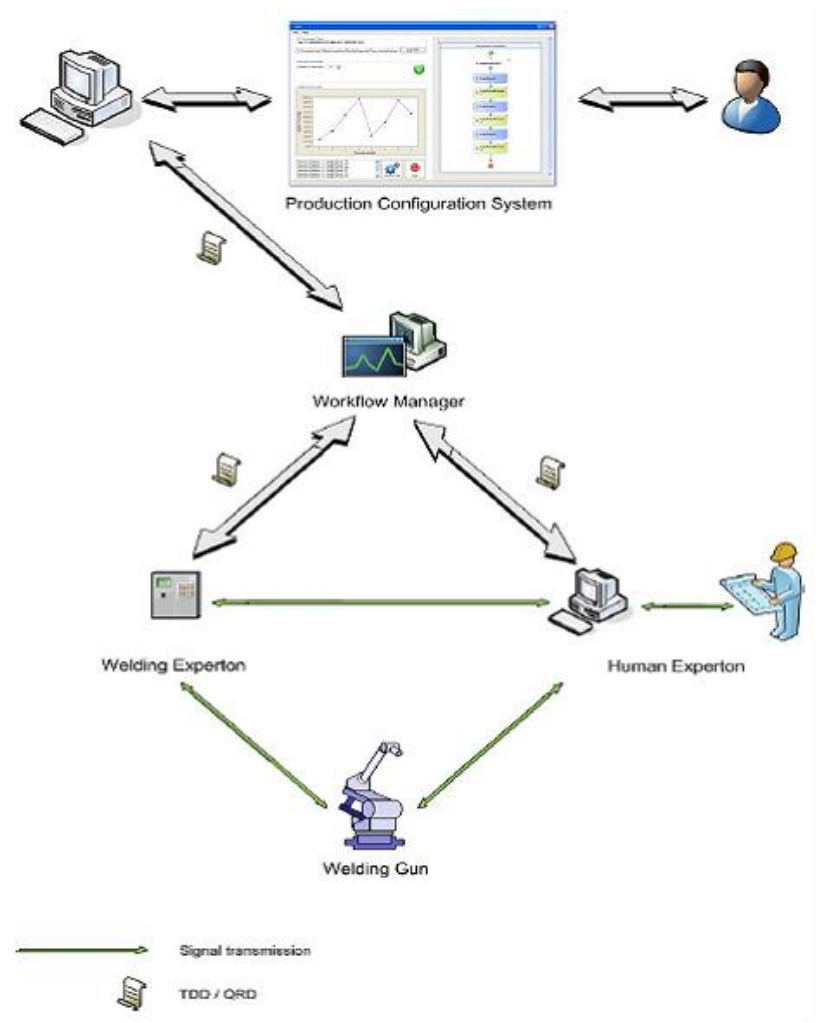

Figure 6. Production Execution System diagram

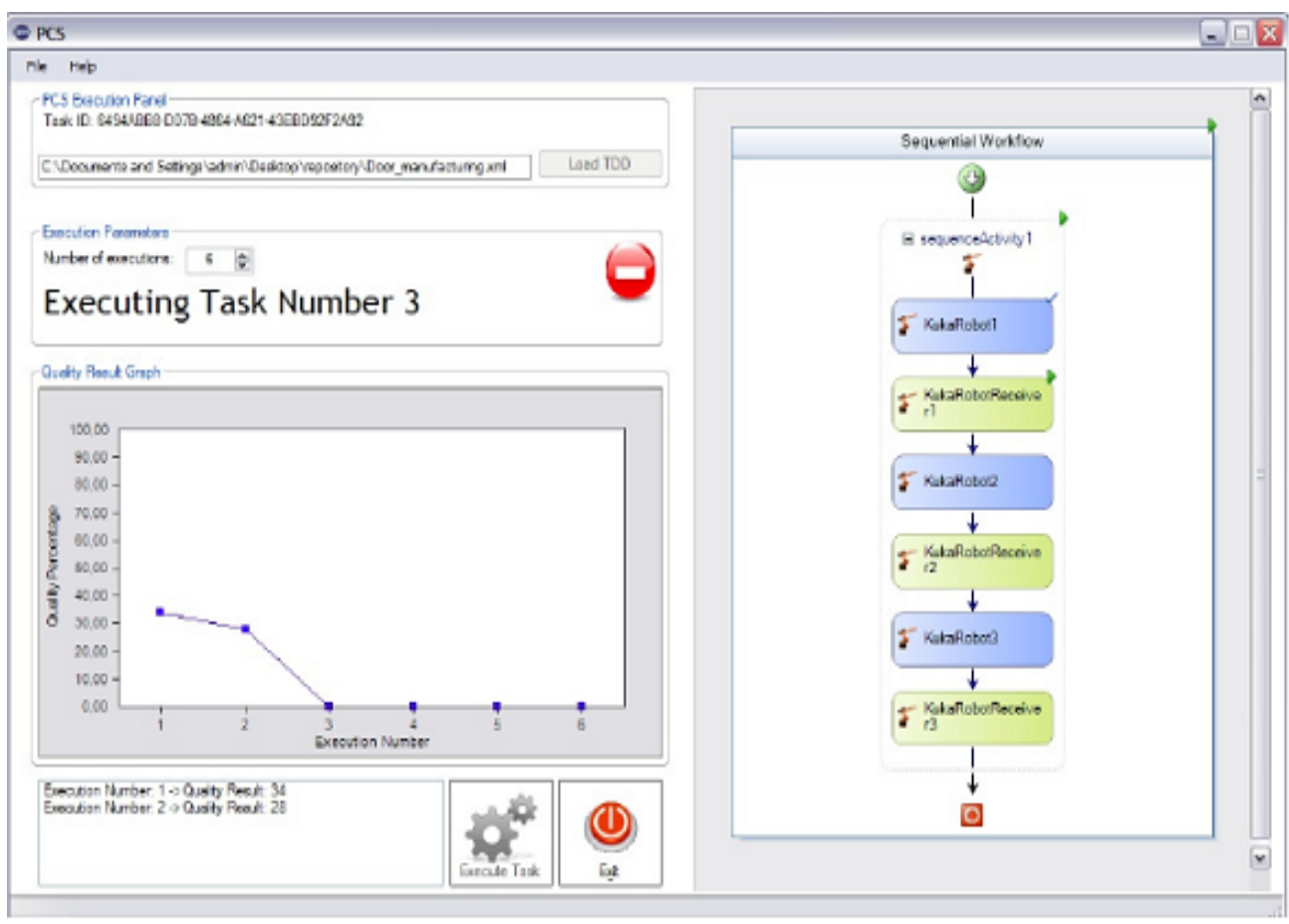

Figure 7. PCS GUI working 
At the same time, the GUI is able to present the quality results, sent back from the WFM to the PES. These results are presented to the end user in a graphical form where the $\mathrm{X}$-axis represents the execution number and the $\mathrm{Y}$-axis represents the quality percentage obtained. After the execution phase, the graphic will contain all the quality results from all the executions and the workflow viewer will display all the activities as finished.

\subsection{Interface to external simulation tools}

The PSS has two possibilities to access data from outside its own area of responsibility: from the PCS knowledge base and from an external simulation tool. The interface to the external simulation tool will be realized via a "simulation manufactron".

The simulation manufactron is based on the universal manufactron and therefore presents to the PSS the I/O interface layer of the universal manufactron. When the PSS requires the services of an external simulation tool, it sends a TDD to the simulation manufactron and gets a QRD in return. The details of unpacking data from the TDD, sending it to the simulation tool, receiving the results of the simulation and packing them into a QRD are all hidden behind the manufactron I/O interface.

Using this approach, the knowledge about how to interpret the TDD data is encapsulated in the simulation manufactron. This encapsulation provides the benefit that any change to the TDD structure is limited in scope. Without it, every time the TDD structure is modified the simulation tool would have to be reprogrammed to understand the new way of data representation.

\section{Implementation}

\subsection{Workflow manager}

The Workflow Manager is a simple console application, with three services to host: Manufactronic Service, Workflow Runtime and Workflow Communication Service. The Workflow Runtime hosts two additional services: tracking and persistence service, which are based upon the standard SQL Server implementation of the Windows Workflow Foundation (WWF). The Workflow Manager can simultaneously execute several tasks.

The Workflow Manager provides an additional Windows Communication Foundation (WCF) service, composed by the following methods: 
- ValidateTask - turns possible the validation of a TDD before the execution

- GetAllWfStatus - get the status of a workflow and its result is given in XML format

- GetInstances - returns all instances according to the filter, which can have one the following values: running, completed or all

- RaiseWorldEvent - provides an external interception possibility in the execution of the workflow

There is also a Workflow Monitoring application, which is an ASP.NET web site. This application communicates with the WfmQm through the Workflow Communication Service and it has read access to the Workflow Tracking and Persistence Database.

On the website is possible to check the running instances, the quality results of the executed tasks and the tracking information (when, which task has been executed, with what result) of the completed and running workflows. Furthermore, on the site is possible to intercept the process of an execution. For example, a WorldEvent can be sent to the WfmQM or a workflow can be aborted if it has a deadlock or an infinite cycle.

Figure 8 depicts an execution example of the Workflow Monitoring application.

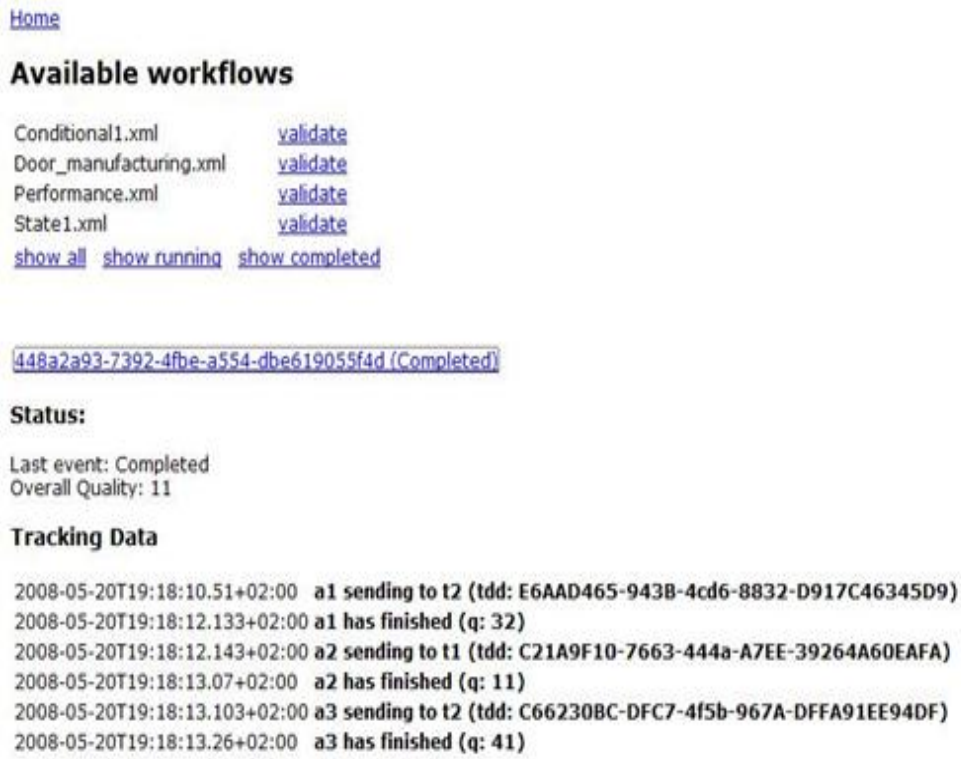

Figure 8. Example of the Workflow Monitoring application 


\subsection{Workflow manager template}

The Workflow Manager Template is embedded into a Task Description Document (TDD). In the manufactronic hierarchy every "instruction" is a TDD. At Workflow, TDD contains one main task, which has the workflow control-flow (executable program) and additional embedded TDDs identified by a TddId, which the controlflow sends to the underlying manufactrons. It is important to emphasize that the TDD is a unique product instance, which follows the rules of the WFM template.

Figure 9 gives an example of a sample control-flow.

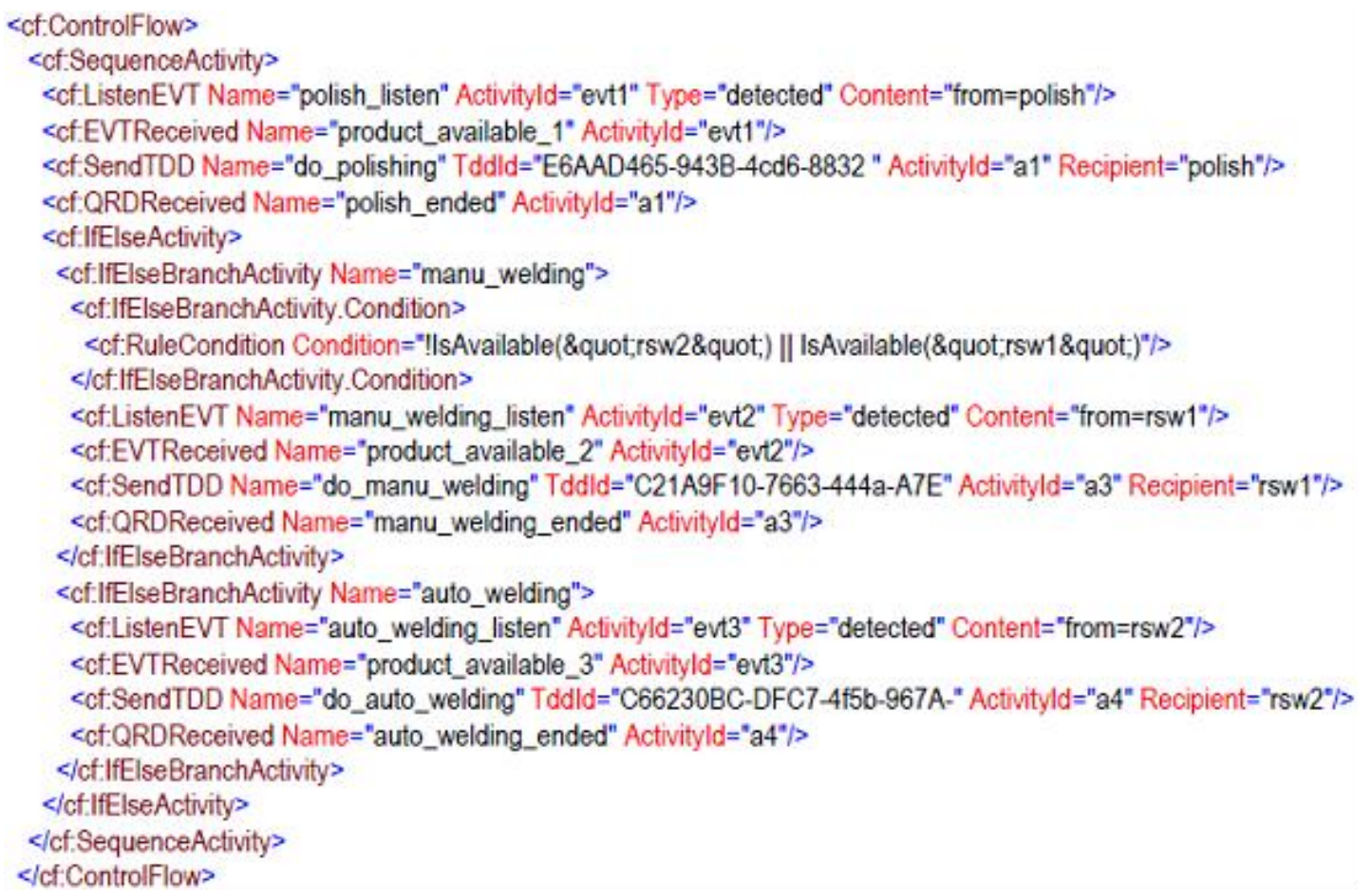

Figure 9. Sample control-flow of the Workflow Manager Template

The cf:ControlFlow is always the root and contains one of the two main containers (Sequence and Sate). The Containers contains compound and simple activities, which can be standard WF activities and Manufactronic primitives too. The template is written in a special Manufactronic dialect, but is similar to eXtensible Object Markup Language (XOML) as much as it can. In the following, sections defining the primitives and their corresponding XOML variant will be presented.

The Sequence Container contains a sequence of activities. It is important to mention that every workflow must have an entry and exit point. In the State Container exists the InitialState and the CompletedSate. Only one state can be activated at a time. The states contain an initialization sequence and an event 
driven activity. The initialization sequence is executed, when the workflow entries into a state activity and at the end it waits for an event, which can trigger the workflow to proceed to a next state. The next state to follow is defined in the SetStateActivity. When the CompletedState is activated the workflow terminates. State machine's path of execution is arbitrary according to the order of events and data. Every execution can differ, contrary to the sequence container, where the execution path is determined beforehand.

Figure 10 defines the template for State Container.

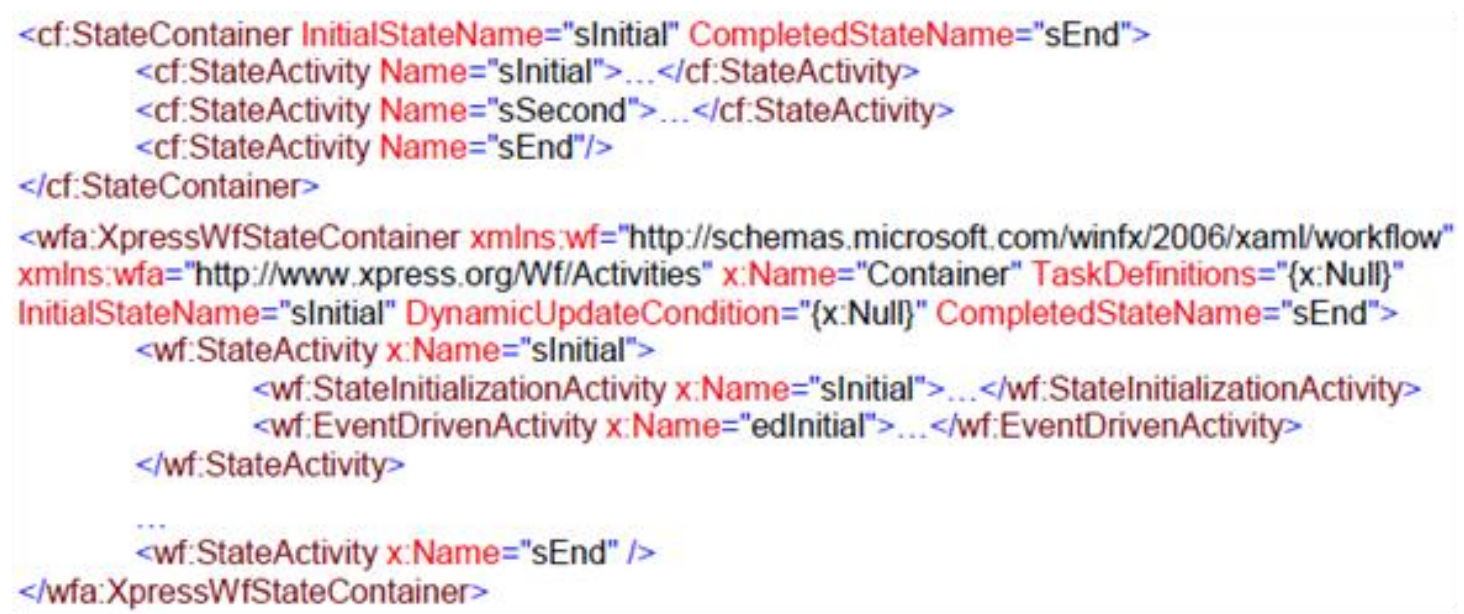

Figure 10. Template definition for State Container

The template includes several workflow primitives, respectively:

- Sequence activity - can contain sequence of activities, which are executed one-by-one. If the execution stops, for example waiting for an event, the workflow won't proceed to the next stop

- Parallel activity - can contain multiple threads. The threads run pseudoparallel, which means that only one activity is executed at a time, but if one thread is blocked the others can proceed freely. It is similar how one processor can run multiple threads in modern operating systems

- List event - notifies the Workflow Runtime that the workflow is waiting for an event. When this event is received by the Workflow Runtime, the corresponding EVTReceived activity is triggered

- Send event - sends an event to a manufactron. It can be paired with an EVTReceived, but it is not mandatory 
- Event received - this activity is waiting for an event from the Workflow Runtime

- Send TDD - sends a TDD to a manufactron. This activity always has a corresponding QRDReceived activity, because it is a requirement by the manufactronic system

- QRD received - this activity is waiting for an event from the Workflow Runtime

- If-else-activity - evaluates a RuleCondition and decides which branch to execute. In this example it checks the availability of manufactrons

- While activity - executes the SequenceActivity in the WhileActivity's body until the RuleCondition evaluates to true

- Generate SendTDD activities - the transformation substitutes the activity with "num" pieces of SendTDD activity. It is used for measure the workflow execution system's performance

- Delay activity - the execution is delayed with TimeOutDuration

\section{Results}

The Manufactronic Networked Approach and all the Production Manufactrons developed and their collaboration were tested to demonstrate their functionality. The existence of several demonstration scenarios encourages potential suppliers to provide their equipment based on the Manufactronic concept. Additionally, potential end users have the possibility to see the Manufactronic networked factory running and can therefore be convinced in an easier way of the manufactronic concept and its advantages.

The following three demonstrators were considered:

- Demonstrator \#1 - quality inspection and process monitoring as well as worker assistance in aeronautic industry

- Demonstrator \#2 - planning process and automatic robot path generation in automotive industry

- Demonstrator \#3 - worker guidance and worker behavior interpretation in automotive industry 


\subsection{Demonstrator \# 1}

This demonstrator is the only one which is directly integrated into an existing and running production line. For that reason, a smooth integration without hampering or slowing down the production is required. The demonstrator intends to fulfil the following objectives:

- Demonstration of the abilities of the riveting Manufactron

- Demonstration of the reliability of the quality assurance system

- Demonstration of closed quality loops for real-time parameter adaptation

Materials of the panels are aluminum and titanium sheets having different thickness. Due to the fact that the demonstrator is completely integrated into a running production line, real panels of an aircraft are used. The costs of one panel or hampering the production are very significant (estimated between $100.000 €$ and $500.000 €)$, therefore, the integration of the system into the production line has to be done very carefully). For setting one rivet, several processes are performed. The usual sequence of setting a rivet is illustrated in Figure 11.

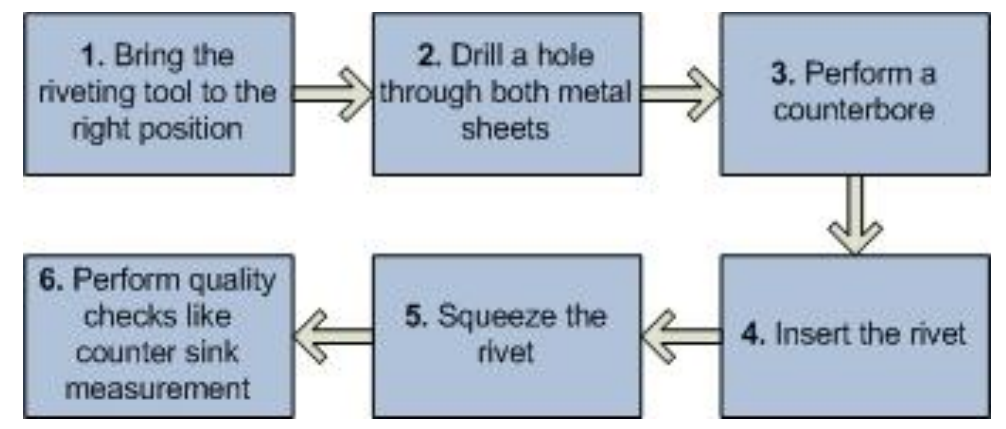

Figure 11 . Sequence of setting a rivet

The demonstrator \#1 provided the following results:

- It demonstrated the $100 \%$ quality assurance of production processes by embedding quality assessment software for the riveting process

- It demonstrated a reactive production with closed-loop control sequences, and the flexible and fault-tolerance reaction by the dynamic adaption of process parameters based on the quality assessment

- It demonstrated the feedback of quality information to CAD data by the visual representation of quality information in virtual CAD environments 
- It demonstrated the feasibility of the Manufactronic approach in the aeronautics sector

\subsection{Demonstrator \#2}

This scenario demonstrates the cooperation of a Handling Manufactron and a Welding Manufactron within an application in the automotive industry. The focus of this scenario is the demonstration of the capabilities of the Handling Manufactron in path planning, automatic path generation and quality assurance. Besides that, this scenario intends to demonstrate the product tracking and production data feedback gathering by the workflow managers.

The scenario consists of three different cars types (station wagon, sedan and coupe) having different shapes. The Figure 12 shows a station wagon.

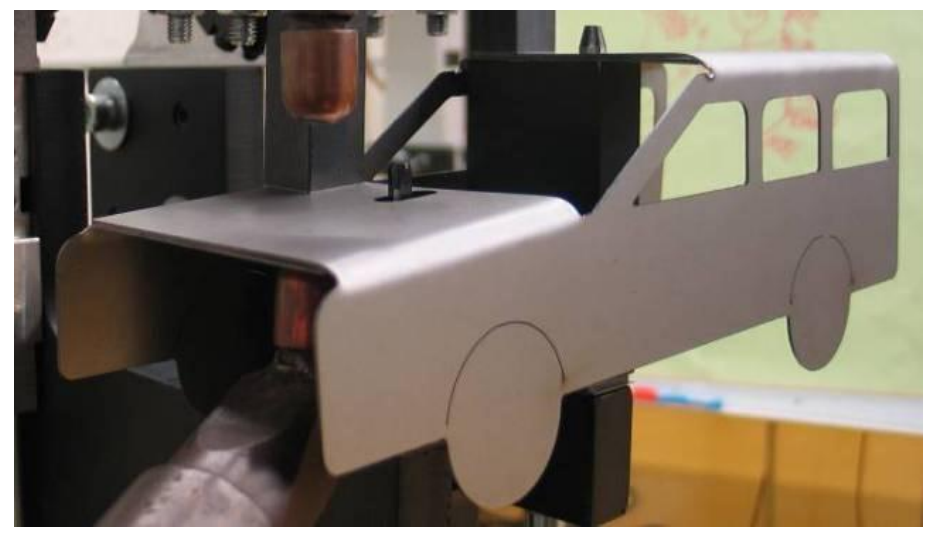

Figure 12. Station wagon

Each product type is built of two metal sheets (left and right side of the car frame). The material and the thickness of the metal sheets do not differ from each other. To weld the different product types, a couple of welding spots are needed. The number and position of the spot differ from type to type. For approaching the different spot locations, a welding gun (mounted on a robot) is used. The insertion and removing of the product from the gripper is done manually.

The demonstrator \#2 provided the following results:

- It demonstrated a reactive production with closed-loop control sequences and the flexible and fault-tolerance reaction by the semi-automated robot path generation 
- It demonstrated the XPRESS approach for a holistic factory-wide process control and monitoring system by gathering quality data of both welding and handling processes

- It contributed to decrease of the ramp-up time for the set-up of production line and the optimization of the product cycle time by the semi-automated robot path generation

- It demonstrated the feasibility of the XRESS concepts in automotive industry

\subsection{Demonstrator \#3}

This demonstrator actually has two different setups. The biggest part is the demonstration of the worker integration into the Manufactronic concept; another setup is the inclusion of Handling Manufactron which focuses on the cooperation of two handling manufactrons based on Cornau robots.

For performing the robot scenarios and the monitoring of the worker sequence (in body shell), cars doors are used. Figure 13 illustrates the production assembly steps of a car door.

The car door body

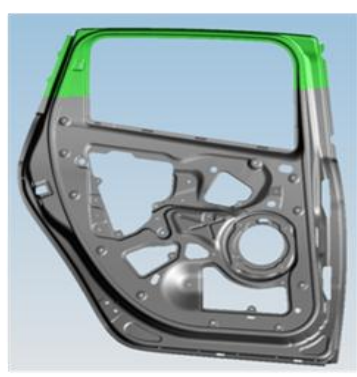

Parts

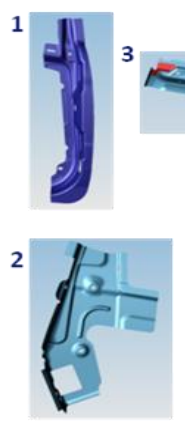

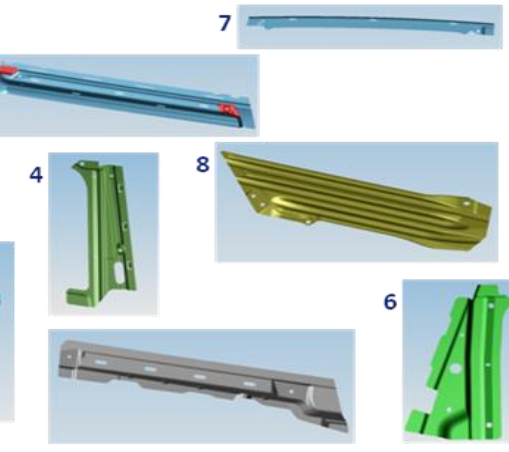

The assembled car door

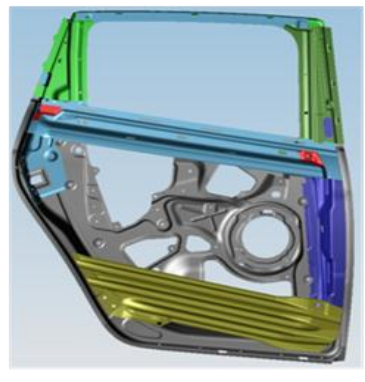

Figure 13. Assembly process of a car door

It is relevant to mention that the materials used in those scenarios are not relevant, because the scenarios do not depend on the material properties. Also the processes (in terms of joining processes) are not that relevant in those scenarios.

The worker integration scenarios provided the following results:

- It demonstrated the $100 \%$ quality assurance of production processes by monitoring the correct sequence of handling tasks by humans 
- It demonstrated the reactive production as well as the flexibility and faulttolerance in production by the identification of wrong components or faulty components using video inspection

- It demonstrated the potential of the XPRESS concept for factory-wide quality data gathering by gathering and assessing quality data of different tasks

- It demonstrated the quality data monitoring by feeding back quality information to the human

The robot cooperation scenario provided the following results:

- It demonstrated the flexible reaction on unexpected production volumes in case of manual production tasks by showing the exchangeability of tasks done by humans and robots

- It demonstrated the reusability of assembly equipment by wrapping a Cornau robot with a Handling Manufactron shell developed for a KUKA robot

\section{Conclusions}

XPRESS meets the challenge to integrate intelligence and flexibility at the "highest" level of the production control system as well as the "lowest" level of the singular machine. The XPRESS manufacturing system integrates a superior cost-efficient production configuration tool in which a complete production line can be reliably simulated as a digital factory. In fact, XPRESS shifts the whole production process from a resource-intensive industry towards knowledge-based and customer-driven approach.

XPRESS provides a structural organization and communication scheme for the field level building on new specialized networking objects, named "Production Manufactrons" which have expert knowledge and capabilities of a specific, basic assembly process. They act as self-responsible specialists in a unit-team to assemble parts under supervision of a unit co-ordinator. Furthermore, XPRESS provides a seamless worker integration in the Manufactronic structure by embedding humans in a system which gives them flexibility and assistance to optimally fulfil a task, while providing standard Manufactron interfaces to the surrounding system. 
To realize this, XPRESS extends the current 2-dimensional organization structure by a 3rd dimension representing the knowledge linking. In the structural organization scheme of the "Holonic Manufacturing" concept, Production Manufactrons can be seen as specialized resource holons with the ability to form knowledge networks. XPRESS also investigates the improvement of the "bureau level", especially concerning production planning and simulation systems. Due to restrictions of available process information, already existing "commercial off the shelf" solutions can only provide a rough planning of production lines, despite the fact that they are very sophisticated software systems. On the contrary, XPRESS proposes the division of these existing systems into simulation and cost estimator (and optimizer) Manufactron and a central configuration Manufactron is added to manage all the information transfer and for the production of workflow managers. Furthermore, with this precise process simulation, optimized mobile agents (Workflow Manufactrons) of the 2nd level are generated automatically for an optimal coordination of the production units in order to produce a specific product variant and for tracking the product along the line.

The radical innovations of the "Manufactronic Networked Factory" are knowledge and responsibility segregation and trans-sectoral process learning in specialist knowledge networks. Assembly units composed of Manufactrons can flexibly perform varying types of complex tasks, whereas today this is limited to a few predefined tasks. By sharing the specific knowledge of each Manufactron in a network, other Manufactrons are able to learn from each other in one production line, but also between different lines as well as different production units. This architecture allows continuous process improvement. Therefore, XPRESS is able to anticipate and to respond to rapidly changing consumer needs, producing high-quality products in adequate quantities while reducing costs.

The concept of Manufactronic networked factory was demonstrated in two representative applications (automotive and aeronautics). XPRESS realized a reactive production with closed-loop control sequences. With this method it was possible to react more flexibly and fault-tolerantly on disturbances and, therefore, the reliability and availability of the production line was increased. With XPRESS it was possible to reach an availability of up to $92 \%$ (state-of-the-art is $87 \%$ ). An important industrial need is also to have a holistic factory-wide process control and monitoring system. XPRESS addressed this issue and proposed an interoperability concept, in which different hardware and software components can be addressed and connected via standard interfaces, enabling a user-friendly, flexible and reliable production concept and also factory-wide process controlling and monitoring 
including weak-point analysis. Feedback to CAD databases in order to optimize the construction of a part is also possible. Finally, the quality assurance system was able to provide a $100 \%$ inline non-destructive quality monitoring. Time needed for the destructive tests was reduced drastically and a reduction of the costs of $30 \%$ $40 \%$ was also reached. Besides that, based in the demonstration scenarios, the ramp-up time for the set-up of production line decreased up to $50 \%$ and the changeover time decreased up to $80 \%$.

\section{Acknowledgments}

This work was carried out with the support from the European Commission under the Sixth Framework Programmer for Research and Technological Development, in the Integrated Project XPRESS (IP026674-2).

\section{References}

Aized, T. (2010). Future Manufacturing. Rijeka: InTech.

Almeida, F., Terra, B., Dias, P., \& Gonçalves, G. (2010). Transport with conveyors in the factory of the future. Proceeding of the IEEE International Conference AQTR, Cluj-Napoca, Romania.

Cheng, F., Chang, C., \& Wu, S. (2004). Development of Holonic manufacturing execution systems. Journal of Intelligent Manufacturing, 15, 253-267. http://dx.doi.org/10.1023/B:JIMS.0000018037.63935.a1

Chituc, C., \& Restive, F. (2009, June). Challenges and trends in distributed manufacturing systems: are wise engineering systems the ultimate answer? Proceeding of the Second International Symposium on Engineering Systems, Massachusetts, USA.

EC (2004). Manufuture: a vision for 2020. http://www.manufuture.org/documents/manufuture_vision_en\%5B1\%5D.pdf. - Accessed July 12th, 2011.

Koestler, A. (1967). The Ghost in the Machine. Ontario: Arcana editions.

Leitão, P. (2009). Agent-based distributed manufacturing control: a state-of-the-art survey. Engineering Applications of Artificial Intelligence, 22(7), 979-991. http://dx.doi.org/10.1016/j.engappai.2008.09.005 
Mekid, S., Pruschek, P., \& Hernandez, J. (2009). Beyond intelligent manufacturing: new generation of flexible intelligent NC machines. Mech Mach Theory, 44(1), 466-476. http://dx.doi.org/10.1016/j. mechmachtheory.2008.03.006

Peschl, M. (2010). Executive summary of XPRESS, http://www.xpressproject.eu/upload/XPRESS_42MonthsPublishableExecutiveSummary.pdf. - Accessed May 17th, 2011.

Pollak, J., Sarveniazi, A., \& Link, N. (2010). Retrieval of process methods from task descriptions and generalized data representations. International Journal of Advanced Manufacturing Technology, 53(5), 1-12.

Prajogo, D., Laosirihongthing, T., Sohal, A., \& Boon, S. (2007). Manufacturing strategies and innovation performance in newly industrialized countries. Industrial $\begin{array}{llll}\text { Management \& Data Systems, 107(1), 52-68. } & \text { i }\end{array}$ http://dx.doi.org/10.1108/02635570710719052

Revilla, J., Christo, C., \& Cardeira, C. (2007). Intelligent manufacturing systems vision about the future. Proceeding of the IEEE International Symposium of Industrial Electronics, Vigo, Spain.

Revilla, J., \& Cadena, M. (2008). Trends in intelligent manufacturing systems. Proceeding of the World Congress on Engineering, London, UK.

Ribeiro, T., \& Gonçalves, G. (2010). Formal methods for reconfigurable assembly systems. Proceeding of the 15th International Conference on Emerging Technologies and Factory Automation, Bilbao, Spain. http://dx.doi.org/10.1109/ETFA.2010.5641248

Sanchez, L., \& Nagly, R. (2001). A review of agile manufacturing systems. International Journal of Productions Research, 39(16), 3561-3600. http://dx.doi.org/10.1080/00207540110068790

Shen, W., \& Norrie, D. (1999). Agent-based systems for intelligent manufacturing: a state-of-the-art survey. Knowledge and Information Systems (KAIS), 1(2), 129156.

Shen, W., Hao, Q., Yoon, H., \& Norrie, D. (2006). Applications of agent-based systems in intelligent manufacturing: an update review. Advanced Engineering Informatics, 20(4), 415-431. http://dx.doi.org/10.1016/j.aei.2006.05.004 
Yang, H., \& Xue, D. (2003). Recent research on developing Web-based manufacturing systems: a review. International Journal of Production Research, 41(15), 3601-3629. http://dx.doi.org/10.1080/0020754031000120014

Journal of Industrial Engineering and Management, 2011 (www.jiem.org)

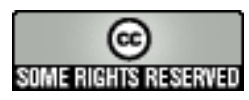

Article's contents are provided on a Attribution-Non Commercial 3.0 Creative commons license. Readers are allowed to copy, distribute and communicate article's contents, provided the author's and Journal of Industrial Engineering and Management's names are included. It must not be used for commercial purposes. To see the complete license contents, please visit http://creativecommons.org/licenses/by-nc/3.0/. 